On the validity of non-Markovian master equation approaches for the entanglement dynamics of two-qubit systems

This article has been downloaded from IOPscience. Please scroll down to see the full text article.

2010 Phys. Scr. 2010014042

(http://iopscience.iop.org/1402-4896/2010/T140/014042)

View the table of contents for this issue, or go to the journal homepage for more

Download details:

IP Address: 147.163.21.159

The article was downloaded on 26/10/2010 at 09:48

Please note that terms and conditions apply. 


\title{
On the validity of non-Markovian master equation approaches for the entanglement dynamics of two-qubit systems
}

\author{
E Ferraro ${ }^{1}$, M Scala ${ }^{1}$, R Migliore $^{2}$ and A Napoli ${ }^{1}$ \\ ${ }^{1}$ CNISM and Dipartimento di Scienze Fisiche ed Astronomiche, Università di Palermo, via Archirafi 36, \\ 90123 Palermo, Italy \\ ${ }^{2}$ CNR-INFM, Research Unit CNISM of Palermo, via Archirafi 36, 90123 Palermo, Italy \\ E-mail: ferraro@ fisica.unipa.it and matteo.scala@fisica.unipa.it
}

Received 29 March 2010

Accepted for publication 16 June 2010

Published 30 September 2010

Online at stacks.iop.org/PhysScr/T140/014042

\begin{abstract}
In the framework of the dissipative dynamics of coupled qubits interacting with independent reservoirs, a comparison between non-Markovian master equation techniques and an exact solution is presented here. We study various regimes in order to find the limits of validity of the Nakajima-Zwanzig and the time-convolutionless master equations in the description of the entanglement dynamics. A comparison between the performances of the concurrence and the negativity as entanglement measures for the system under study is also presented.
\end{abstract}

PACS numbers: 42.50.Lc, 03.65.Yz

\section{Introduction}

The problem of the entanglement between two two-level systems interacting with thermal baths has been given a lot of attention in recent years [1-8] because of its importance both from a fundamental and from an applicative point of view. The open system dynamics is usually approached by means of master equation methods, both for the Markovian and the non-Markovian cases. Concerning the non-Markovian dynamics, in which the memory effects in the reservoirs become important, two possible methods are generally used, namely the Nakajima-Zwanzig (NZ) [9, 10] and the time-convolutionless (TCL) [11] approaches. The first one leads to an integro-differential evolution equation for the system density operator, so that its time derivative depends on its previous history, whereas the second one leads to a generalized master equation that is local in time.

In a previous paper, we studied the validity of the two approaches for a system whose dynamics, in some cases, turns out to be exactly solvable [12]. The system investigated is composed of two interacting qubits, each one coupled to its own bosonic reservoir, and its Hamiltonian is given by

$$
H=H_{0}+H_{\mathrm{I}},
$$

where

$$
H_{0}=\frac{\omega_{0}}{2} \sigma_{z}^{(1)}+\frac{\omega_{0}}{2} \sigma_{z}^{(2)}+\sum_{j=1,2} \sum_{k} \omega_{k}^{(j)} b_{k}^{(j) \dagger} b_{k}^{(j)}
$$

and

$$
\begin{aligned}
H_{\mathrm{I}}= & \Omega\left(\sigma_{+}^{(1)} \sigma_{-}^{(2)}+\sigma_{-}^{(1)} \sigma_{+}^{(2)}\right) \\
& +\sum_{j=1,2}\left(\sigma_{+}^{(j)} \sum_{k} g_{k}^{(j)} b_{k}^{(j)}+\sigma_{-}^{(j)} \sum_{k} g_{k}^{(j) *} b_{k}^{(j) \dagger}\right) .
\end{aligned}
$$

In equation (2), the transition frequencies of the two two-level systems, supposed to be coincident for simplicity, are indicated by $\omega_{0}$, whereas $\sigma_{z}^{(j)}(j=1,2)$ denotes the Pauli operator describing the $j$ th subsystem. The two independent bosonic baths are characterized by proper frequencies $\omega_{k}^{(j)}$, with $b_{k}^{(j) \dagger}$ and $b_{k}^{(j)}$ being correspondingly the creation and annihilation bosonic operators. The interaction term explicitly given by equation (3) includes both the direct interaction between the two qubits, characterized by the coupling constant $\Omega$, and the interaction between each qubit and its respective bosonic bath. The coupling constants $g_{k}^{(j)}$ characterized the intensity of the coupling between the $j$ th two-level system and the $k$ th bosonic mode of its bath. Finally, $\sigma_{ \pm}^{(j)} \equiv \frac{1}{2}\left(\sigma_{x}^{(j)} \pm i \sigma_{y}^{(j)}\right)$ are, as usual, the lowering and raising Pauli operators. 
In [12], comparing the exact solution with the ones obtained by the NZ and TCL methods, we found that the TCL approach works better than the NZ approach. This is in contrast to what one would expect according to his own intuition and similar to what happens in other physical contexts [13], at least for the populations and in correspondence to a wide range of parameters. Moreover, we found that the NZ approach may violate the positivity condition on the map describing the time evolution, since for certain values of the reservoir parameters, it can predict negative populations.

In this paper, we extend the comparison between the exact and the approximated approaches by focusing our attention on the entanglement dynamics in the two-qubit system. In particular we study the time evolution of both the concurrence and the negativity. The aim of our analysis is twofold. On the one hand, it is important to verify whether the range of validity is still wider for the TCL approach also for the study of the entanglement dynamics. On the other hand, we would like to get some information on which entanglement measure is more reliable when one has to do with approximated solutions. For example, this information might turn out to be useful in the analysis of the nonzero temperature dynamics of our systems, for which an exact solution is not available. We will see that the TCL approach works better than NZ also for the entanglement dynamics and that for our problem the time evolution of the concurrence is closer to the exact solutions than the negativity.

The results will be presented in the following section where two different entanglement measures for the bipartite system, namely the concurrence and the negativity, will be calculated for different values of the reservoir memory times. In the last section, some conclusive remarks are given.

\section{Entanglement dynamics}

The exact solution of the dynamics for the system under investigation can be obtained exploiting the conservation of the total number of excitations corresponding to the number operator

$$
\hat{N}=\sum_{j=1,2} \sigma_{+}^{(j)} \sigma_{-}^{(j)}+\sum_{j=1,2} \sum_{k} b_{k}^{(j) \dagger} b_{k}^{(j)} .
$$

In particular, if the two-qubit system starts from a state with one excitation, whereas both the two baths are in their respective vacuum state, the state of the total system at a generic time $t$ can be written as

$$
\begin{aligned}
|\psi(t)\rangle= & (a(t)|10\rangle+b(t)|01\rangle)\left|0_{k}^{(1)} 0_{k}^{(2)}\right\rangle+|00\rangle \\
& \times\left(\sum_{k} c_{k}^{(1)}(t)\left|1_{k}^{(1)} 0_{k}^{(2)}\right\rangle+\sum_{k} c_{k}^{(2)}(t)\left|0_{k}^{(1)} 1_{k}^{(2)}\right\rangle\right) .
\end{aligned}
$$

In the interaction picture with respect to $H_{0}$, the probability amplitudes $a(t)$ and $b(t)$ satisfy the following coupled differential equations:

$$
\left\{\begin{array}{l}
\dot{a}(t)=-\mathrm{i} \Omega b(t)-\mathrm{i} \int_{0}^{t} a\left(t^{\prime}\right) f_{1}\left(t-t^{\prime}\right) \mathrm{d} t^{\prime}, \\
\dot{b}(t)=-\mathrm{i} \Omega a(t)-\mathrm{i} \int_{0}^{t} b\left(t^{\prime}\right) f_{2}\left(t-t^{\prime}\right) \mathrm{d} t^{\prime},
\end{array}\right.
$$

where the kernel $f_{j}\left(t-t^{\prime}\right)$ is the correlation function, which in the continuum limit assumes the form

$$
f_{j}\left(t-t^{\prime}\right)=\int_{0}^{+\infty} \mathrm{d} \omega J_{j}(\omega) \mathrm{e}^{\mathrm{i}\left(\omega_{0}-\omega\right)\left(t-t^{\prime}\right)},
$$

with $J_{j}(\omega)$ being the spectral density of the $j$ th bath. In the following, we assume that each qubit interacts resonantly with a reservoir with Lorentzian spectral density:

$$
J_{1}(\omega)=J_{2}(\omega) \equiv J(\omega)=\frac{1}{2 \pi} \frac{\gamma \lambda^{2}}{\left(\omega_{0}-\omega\right)^{2}+\lambda^{2}},
$$

where $\gamma$ is a parameter that in the Markovian limit coincides with the system decay rate and $\lambda$ is the reservoir bandwidth.

On the other hand, the same problem can be treated by means of the projection operator techniques [13], which in the second-order approximation leads to the following NZ master equation for the reduced density operator of the two-qubit system:

$$
\begin{aligned}
\frac{\mathrm{d}}{\mathrm{d} t} \rho_{\mathrm{S}}(t)= & -\mathrm{i}\left[H_{\mathrm{I}}^{(s)}, \rho_{\mathrm{S}}(0)\right] \\
& -\int_{0}^{t} \mathrm{~d} t^{\prime} \operatorname{Tr}_{\mathrm{B}}\left\{\left[H_{\mathrm{I}}(t),\left[H_{\mathrm{I}}\left(t^{\prime}\right), \rho_{\mathrm{S}}\left(t^{\prime}\right) \otimes \rho_{\mathrm{B}}\right]\right]\right\}
\end{aligned}
$$

The second-order TCL master equation can be obtained from the latter equation by substituting $\rho_{\mathrm{S}}\left(t^{\prime}\right)$ with $\rho_{\mathrm{S}}(t)$.

In [12], we provided the solution for the system in equation (6) and for the NZ and TCL master equations. In that paper, we focused our attention on the time evolution of the populations in order to find the range of validity of the approximated NZ and TCL approaches. Here we look at the entanglement dynamics between the two qubits by calculating two different entanglement measures, namely the concurrence and the negativity functions [14]. The first one is defined as $C(t)=\max \left(0, \sqrt{\lambda_{1}(t)}-\sqrt{\lambda_{2}(t)}-\sqrt{\lambda_{3}(t)}-\sqrt{\lambda_{4}(t)}\right)$, where $\left\{\lambda_{i}(t)\right\}$ are the eigenvalues of the matrix $R(t)=\rho(t) \tilde{\rho}(t)$, with $\tilde{\rho}(t)=\sigma_{y} \otimes \sigma_{y} \rho^{*}(t) \sigma_{y} \otimes \sigma_{y}, \sigma_{y}$ being the Pauli matrix and $\rho(t)$ the density matrix representing the quantum state of the system. The negativity is given instead by $\mathcal{N}(t)=$ $\sum_{i}\left(\left|a_{i}\right|-a_{i}\right) / 2$, with $a_{i}$ being the $i$ th eigenvalue of the partial transpose of the density matrix with respect to the second qubit. Let us suppose that the two baths are in a thermal state at zero temperature, and the two qubits are initially in the Bell state $(1 / \sqrt{2})(|10\rangle-|01\rangle)$. Figures $1-6$ show the comparison between the exact solution and the approximated ones in correspondence to three different values of $\lambda$. The value $\lambda=10 \gamma$ corresponds to the Markovian regime, while $\lambda=\gamma$ and $\lambda=0.01 \gamma$ correspond, respectively, to a weakly and a strongly non-Markovian regime.

In the Markovian limit, as shown by the concurrence in figure 1 and the negativity in figure 2, we can see perfect agreement between the exact and the TCL solution and quantitative disagreement of the NZ approach, which anyway predicts a very similar qualitative behaviour for the entanglement decay.

In the weakly non-Markovian regime, as shown in figures 3 and 4, we observe also qualitative disagreement between the NZ predictions and the exact dynamics, which is still in agreement with the TCL solution. 


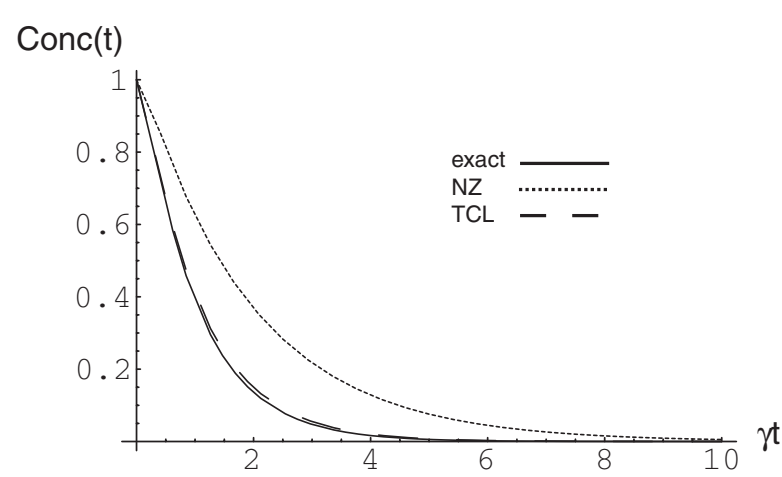

Figure 1. Concurrence function for a system initially prepared in the Bell state $1 / \sqrt{2}(|10\rangle-|01\rangle)$. The width of the Lorentzian spectral density is $\lambda=10 \gamma$; the strength of the coupling constant between the two qubits is $\Omega=0.001 \gamma$.

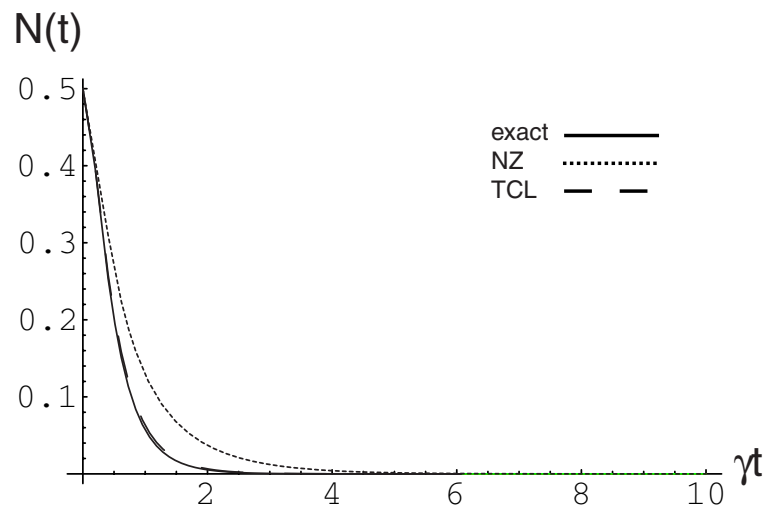

Figure 2. Negativity for a system initially prepared in the Bell state $1 / \sqrt{2}(|10\rangle-|01\rangle)$. The width of the Lorentzian spectral density is $\lambda=10 \gamma$; the strength of the coupling constant between the two qubits is $\Omega=0.001 \gamma$.

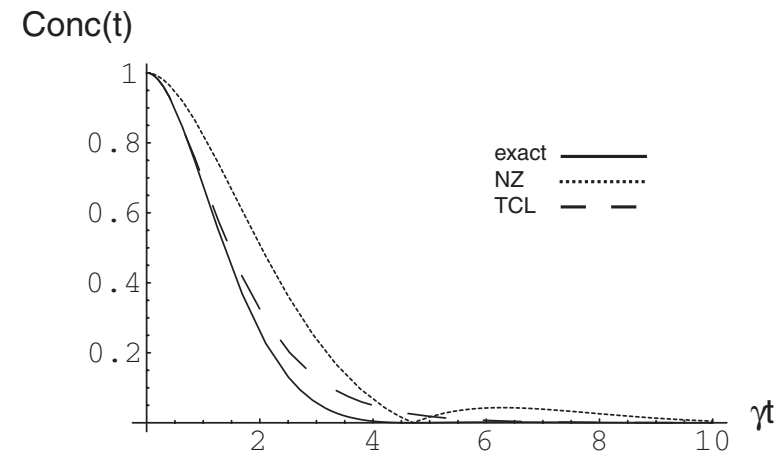

Figure 3. Concurrence function for a system initially prepared in the Bell state $1 / \sqrt{2}(|10\rangle-|01\rangle)$. The width of the Lorentzian spectral density is $\lambda=\gamma$; the strength of the coupling constant between the two qubits is $\Omega=0.001 \gamma$.

Finally, in the strongly non-Markovian regime (see figures 5 and 6), we can see that the TCL approach also fails in the description of the entanglement dynamics, since it is not able to describe the oscillatory behaviour of the exact solution. Concerning the NZ solution, we note that oscillations are present, which mirror the oscillations in the population dynamics under the same conditions [12]. Nevertheless, it is worth noting that the behaviour of the concurrence presents cusps in the regions close to zero, which is rather



Figure 4. Negativity for a system initially prepared in the Bell state $1 / \sqrt{2}(|10\rangle-|01\rangle)$. The width of the Lorentzian spectral density is $\lambda=\gamma$; the strength of the coupling constant between the two qubits is $\Omega=0.001 \gamma$.

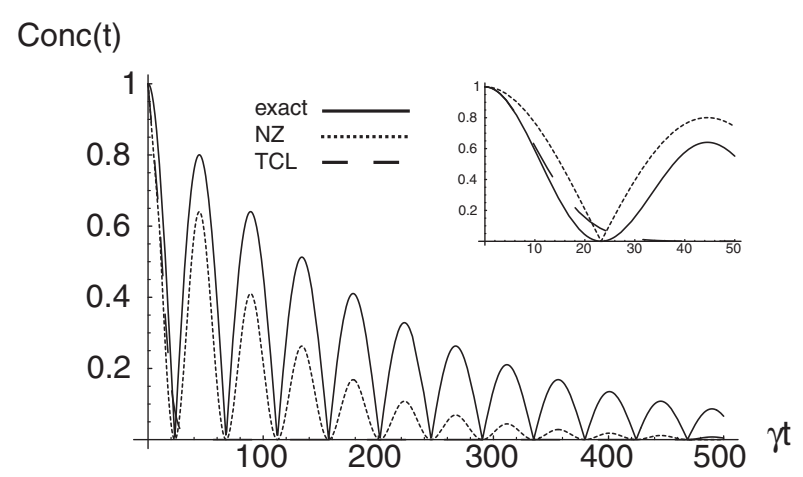

Figure 5. Concurrence function for a system initially prepared in the Bell state $1 / \sqrt{2}(|10\rangle-|01\rangle)$. The width of the Lorentzian spectral density is $\lambda=0.01 \gamma$; the strength of the coupling constant between the two qubits is $\Omega=0.001 \gamma$. The inset shows the short-time dynamics.

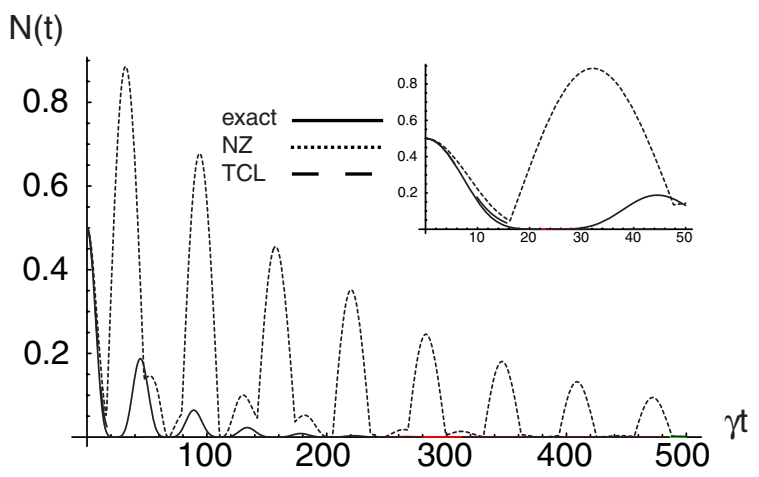

Figure 6. Negativity for a system initially prepared in the Bell state $1 / \sqrt{2}(|10\rangle-|01\rangle)$. The width of the Lorentzian spectral density is $\lambda=0.01 \gamma$; the strength of the coupling constant between the two qubits is $\Omega=0.001 \gamma$. The inset shows the short-time dynamics.

different from the smooth behaviour characterizing the exact solution.

It is worth discussing the failure of the NZ approach, since it can be directly connected to the lack of positivity of the map generated by equation (9). Indeed, in [12] we have seen that, for the cases $\lambda=\gamma$ and $\lambda=0.01 \gamma$, the solution for the populations of the states $|10\rangle$ and $|01\rangle$ can reach 
negative values, so that they cannot represent physical states anymore. The negative values of these populations also affect the coherences between these two states and consequently they affect the concurrence and the negativity.

From our analysis, another point to be discussed emerges. Indeed, for the cases examined, it is apparent that the concurrence works better than the negativity for the description of the entanglement dynamics in the NZ approach. This can be explained by looking at the structure of the system density matrix at any time instant $t$, which, starting from the Bell state under study, is given by

$$
\rho(t)=\left(\begin{array}{cccc}
\rho_{00,00} & 0 & 0 & 0 \\
0 & \rho_{01,01} & \rho_{01,10} & 0 \\
0 & \rho_{10,01} & \rho_{10,10} & 0 \\
0 & 0 & 0 & 0
\end{array}\right) .
$$

From this structure it is obvious that the concurrence depends only on the coherences, since it can be shown that it is equal to $\mathcal{C}(t)=2\left|\rho_{01,10}\right|$. The negativity depends instead on the partial transpose of the density matrix with respect to the second qubit, i.e.

$$
\rho^{T_{2}}(t)=\left(\begin{array}{cccc}
\rho_{00,00} & 0 & 0 & \rho_{01,10} \\
0 & \rho_{01,01} & 0 & 0 \\
0 & 0 & \rho_{10,10} & 0 \\
\rho_{10,01} & 0 & 0 & 0
\end{array}\right) .
$$

Therefore, whereas the concurrence is sensitive to the coherences only, the negativity can be sensibly affected by the negative values of the populations $\rho_{01,01}$ and $\rho_{10,10}$, which are two of the eigenvalues of $\rho^{T_{2}}(t)$, causing a larger deviation of the NZ from the exact negativity.

\section{Conclusive remarks}

In this paper, we have presented a systematic comparison between different non-Markovian master equation approaches to the entanglement dynamics of two coupled qubits interacting with independent reservoirs. In particular, by exploiting a model that can be exactly solved, we have found the range of validity of the NZ and TCL master equations and we have seen that their failure can be directly linked to their failure in the description of the populations of the quantum states. Our results bring highlight that, generally speaking, the TCL approach has to be preferred to the NZ method when we are interested in the entanglement dynamics. The analysis we have developed suggests, in addition, that when approximated methods are used, the concurrence function works better than the negativity for a description of the entanglement evolution of our system. This information should be taken into account for a possible extension of the work to the nonzero temperature case, for which an exact solution is not available. We believe that our analysis can be of importance for a deeper understanding of the non-Markovian master equation techniques, which are nowadays of increasing importance in the description of the dynamics of quantum systems.

\section{References}

[1] Das S and Agarwal G S 2009 J. Phys. B: At. Mol. Opt. Phys. 42205502

[2] Sinaysky I, Petruccione F and Burgarth D 2008 Phys. Rev. A 78062301

[3] Quiroga L, Rodríguez F J, Ramírez M E and París R 2007 Phys. Rev. A $\mathbf{7 5} 032308$

[4] Maniscalco S, Francica F, Zaffino R L, Lo Gullo N and Plastina F 2008 Phys. Rev. Lett. 100090503

[5] Mazzola L, Maniscalco S, Piilo J, Suominen K-A and Garraway B M 2009 Phys. Rev. A 80012104

[6] Yu T and Eberly J H 2010 Opt. Commun. 283676

[7] Scala M, Migliore R and Messina A 2008 J. Phys. A: Math. Theor. 41435304

[8] Sinayskiy I, Ferraro E, Napoli A, Messina A and Petruccione F 2009 J. Phys. A: Math. Theor. 42485301

[9] Nakajima S 1958 Prog. Theor. Phys. 70948

[10] Zwanzig R 1960 J. Chem. Phys. 331338

[11] Chaturvedi S and Shibata F 1979 Z. Phys. B 35297

[12] Ferraro E, Scala M, Migliore R and Napoli A 2009 Phys. Rev. A 80042112

[13] Breuer H-P and Petruccione F 2002 The Theory of Open Quantum Systems (Oxford: Oxford University Press)

[14] Horodecki R, Horodecki P, Horodecki M and Horodecki K 2009 Rev. Mod. Phys. 81 865-942 